P. O. Box 520875, Biscayne Annex, Miami, Fla. 33152 (USA).

38. This work was supported by Research Grant AIl0422 and Training Grant Al00445 from the National Institute of Allergy and Infectious Diseases, and Genetic Center Grant GM19443 from the National Institute of General Medical Sciences, National Institutes of Health, United States Public Health
Service and a grant from the Goldhirsch Foundation, New York, N. Y. 39. Requests for reprints should be addressed to: N. M. Zaldivar, M.D. Department of Pediatrics, Division of Infectious Diseases and Immunology, P.O. Box 520875, Biscayne Annex, Miami, Fla. 33152 (USA).

40. Accepted for publication March 10, 1975.

\title{
The Effect of Increased Pulmonary Blood Flow on the Pulmonary Vascular Bed in Pigs
}

\author{
BEAT FRIEDLI, ${ }^{17)}$ GERALDINE KENT, AND B. S. LANGFORD KIDD \\ WITH THE TECHNICAL ASSISTANCE OF M. LUIDE AND F. HAMILTON \\ Department of Cardiology and Research Institute, The Hospital for Sick Children, Toronto, Ontario, Canada
}

Extract

Increased pulmonary blood flow was produced in 1-month-old piglets by means of left pneumonectomy, arteriovenous fistulas in the neck, and a combination of both. Physiologic and histologic studies of the pulmonary vascular bed were done 1-9 months after operation.

A progressive, moderate increase in pulmonary artery (PA) pressure was observed, especially between 1 and 6 months after surgery. This was flow related, i.e., the group with the highest flow (pneumonectomy plus fistula) was found to have the most prominent increase in pressure. Mean PA pressure at 6 months was $28.7 \pm$ $0.07 \mathrm{~mm} \mathrm{Hg}$ in this group, vs $24.4 \pm 0.48 \mathrm{~mm} \mathrm{Hg}$ in the group with pneumonectomy alone and $17.2 \pm 0.48 \mathrm{~mm} \mathrm{Hg}$ in controls $(P<$ 0.01 ). The pressure response to hypoxia in pigs with high pulmonary blood flow was not different from that found in control animals.

Histologic studies revealed that small arteries and arterioles of pigs with high pulmonary blood flow had a decreased relative wall thickness because of dilation up to 6 months follow-up. This was flow related, the group with the highest flow having the lowest wall thickness to vessel diameter ratio; relative wall thickness (in percentage of the vessel diameter) at 6 months was $6.1 \pm 0.44 \%$ in pigs with pneumonectomy plus fistula, vs $\mathbf{9 . 6} \pm \mathbf{0 . 4 0} \%$ in the group with pneumonectomy alone and $11.2 \pm 0.61 \%$ in controls $(P<$ $0.01)$. In the group with the highest flow, thick walled arterioles appeared at 9 months follow-up, scattered among dilated ones; mean medial wall thickness in these animals increased significantly between 6 and 9 months after operation, ranging from $6.1 \pm 0.44 \%$ to $11.3 \pm 0.73 \%(P<\mathbf{0 . 0 1})$.

In five animals with high flow, the right $\mathrm{PA}$ ( main branch) showed patchy intimal thickening, small cystic spaces filled with mucopolysaccharides in the media, and muscular hypertrophy.

\section{Speculation}

Some changes usually seen in pulmonary hypertension were produced in this model by high pulmonary blood flow in the presence of only mild to moderate elevation of PA pressure. The dilation observed in the pulmonary arteries and arterioles may have been a pathogenetic factor; indeed, it is likely that wall tension rather than intravascular pressure induces vascular changes; wall tension (according to Laplace's law) depends on vessel diameter as well as intravascular pressure. Therefore, in a dilated vascular bed, wall tension will increase considerably with only moderate increase in PA pressure, inducing changes usually seen with more severe degrees of pulmonary hypertension.

Pulmonary vascular disease remains a serious complication of congenital heart disease with a left-to-right shunt. Little is known about the pathogenesis of this condition, but three factors are believed to be involved: increased pulmonary blood flow, a raised pulmonary artery pressure, and high left atrial pressure.

Experimental models used to study pulmonary vascular disease often involve anastomoses between aorta and pulmonary artery, whereby two if not all three of the above mentioned factors are produced $(1,4)$.

In an attempt to identify changes induced by high flow alone, we have created "obligatory" shunts in piglets $1-2$ months old; this was obtained by unilateral pneumonectorny, creation of systemic arteriovenous fistulae, and a combination thereof.

\section{METHODS}

Three litters of piglets were used. Each litter was divided into four groups: two pigs were not subjected to surgery (controls); two underwent left pneumonectomy at 4 weeks of age with no other operation; two had pneumonectomy at the same age, followed by creation of arteriovenous (A-V) fistulas at 4-6 weeks of age; two had A-V fistulas alone. Thus, there were eight animals in each group. A-V fistulas were created between the carotid artery and the internal jugular vein. Incisions $2 \mathrm{~cm}$ long were made in these vessels and a side-to-side anastomosis created by continuous sutures. A unilateral fistula was made in pigs of the first litter, and bilateral anastomoses in those of the second and third litters. One litter was followed up for 1-3 months, the second for 6 months, and the third for 9 months. Subsequently, the pigs were killed for histologic study.

Hemodynamic studies were repeated at 1, 3, 6, and 9 months for as long as the animals were alive. 


\section{HEMODYNAMIC STUDY}

Pigs were anesthetized with pentobarbital $(20 \mathrm{mg} / \mathrm{kg})$. A Swan-Ganz balloon-tipped catheter was floated into the pulmonary artery from a peripheral vein and a Teflon catheter placed into a femoral or axillary artery to measure systemic pressure and arterial blood gases.

Small pigs were ventilated on a Palmer pump, larger animals on a Harvard respirator. Blood gases were monitored by an Instrumentation Laboratory blood gas analyzer. An Electronics for Medicine recorder was used to record pressures in the pulmonary artery and systemic artery. An estimate of the left atrial pressure was obtained by measuring capillary wedge pressure; this could be achieved easily by slightly inflating the balloon of the Swan-Ganz catheter.

Cardiac output was measured by dye dilution curves and the direct Fick principle. A Kipp oxymeter was used to measure oxygen content, and expired air was analyzed on a DCL Servomex oxygen analyzer.

Baseline Study. Each pig was ventilated with room air to have a $\mathrm{Pa}_{\mathrm{O}_{2}}$ of $75-95 \mathrm{~mm} \mathrm{Hg}$; $\mathrm{pH}$ ranged from 7.44 to 7.55 (Table 2). PA pressure, systemic pressure, capillary wedge pressure, and cardiac output were measured. Cardiac index was calculated using Meeh's constant relating weight to body surface area in pigs (12).

Hypoxia. Each animal was then ventilated with $5 \%$ oxygen in nitrogen to induce a hypoxic pulmonary vascular response. After 3 min, when $\mathrm{Pa}_{\mathrm{O}}$, had dropped to $20-30 \mathrm{~mm} \mathrm{Hg}$, pressure and output measurements, as outlined above, were taken again.

\section{HISTOLOGIC STUDY}

After the last hemodynamic study, each pig was killed by giving intravenous potassium chloride in a dosage sufficient to cause cardiac arrest. The right lung was rapidly removed and inflated with $5 \%$ buffered formalin through the bronchus under a pressure head of $50 \mathrm{~cm} \mathrm{H}_{2} \mathrm{O}$. After $48 \mathrm{hr}$, sections were taken from the upper and lower lobes centrally and peripherally, and from the right pulmonary artery at its site of entry into the pulmonary parenchyma. Histologic sections were made using hematoxylineosin, elastic, periodic acid-Schiff, alcian blue, and colloidal iron stains.

Relative medial wall thickness of arterioles and small arteries, $20-100 \mu$ in diameter was measured according to the method of Waagenvoort (14). Twenty such measurements were made for each pig, 10 in the upper and 10 in the lower lobe.

\section{RESULTS}

In three pigs (two without and one with pneumonectomy) the arteriovenous fistulas closed spontaneously after 1-2 months; these animals were eliminated from the study. Each shunt in all other animals remained open throughout the follow-up. This was demonstrated by presence of a continuous murmur in the neck and dye dilution curves showing evidence of a left-to-right shunt.

\section{PULMONARY BLOOD FLOW}

Cardiac output (Table 1) was increased by $73 \%$ in pigs with $\mathrm{A}-\mathrm{V}$ fistulae; the increase was most marked in the 3 month follow-up study. Left pneumonectomy increases the blood flow through the right lung by approximately $75 \%$. Therefore, in pigs with pneumonectomy and A-V fistula, the blood flow to the remaining lung was increased by a factor slightly greater than 3 .

\section{PULMONARY ARTERY PRESSURES (FIG. 1, TABLE 2 )}

PA pressures were low in control animals and did not change significantly during the follow-up study (i.e., from the ages of $2-10$ months). All operated animals showed a progressive increase in PA pressures, particularly up to 6 months postoperatively; little further increase occurred between 6 and 9 months. The rise in pressure was especially marked in pigs with both pneumonectomy and shunt, where the mean PA pressure was already significantly different from the control at 1 month after operation $(P<0.05)$. PA pressures in pigs with either pneumonectomy or A-V fistula alone increased to a lesser degree. The mean pressure was different from the control at the 3-month level and later $(P<0.01)$.

\section{CAPILLARY WEDGE PRESSURES}

These remained within normal limits at all times. Mean capillary wedge pressures showed a tendency to be higher in animals with A-V fistulas than in those without, but this difference was not statistically significant.

\section{RESISTANCE}

Pulmonary arteriolar resistance was increased slightly in pigs with pneumonectomy. This was apparent from the lst month postoperatively in those without an A-V fistula, and at 6 months only in those with both pneumonectomy and shunt (Table 2).

\section{REACTION TO HYPOXIA}

There was a marked pressure response to hypoxia in all pigs. The increase in mean PA pressure persisted throughout the follow-up and was even slightly more marked at 6 and 9 months. A somewhat higher mean PA pressure in response to hypoxia was reached in pigs with pneumonectomy and $\mathrm{A}-\mathrm{V}$ fistula and also in those with pneumonectomy alone. However, the percentage increase above resting value was similar in all animals. Arteriolar

Table 1. Cardiac output and index at rest, breathing room air $\left(\mathrm{FiO}_{2} 21 \%\right)$, and during hypoxia $\left(\mathrm{FiO}_{2} 5 \%\right)(\text { liters per minute })^{1}$

\begin{tabular}{|c|c|c|c|c|c|c|c|c|}
\hline & \multicolumn{2}{|c|}{1 month } & \multicolumn{2}{|c|}{3 months } & \multicolumn{2}{|c|}{6 months } & \multicolumn{2}{|c|}{9 months } \\
\hline & Output & Index & Output & Index & Output & Index & Output & Index \\
\hline \multicolumn{9}{|l|}{ Control } \\
\hline $\mathrm{FiO}_{2}(21 \%)$ & $2.0 \pm 0.07$ & $4.2 \pm 0.37$ & $3.8 \pm 0.14$ & $5.1 \pm 0.32$ & $4.9 \pm 0.46$ & $3.5 \pm 0.30$ & $10.4 \pm 0.90$ & $5.4 \pm 0.10$ \\
\hline $\mathrm{FiO}_{2}(5 \%)$ & $2.6 \pm 0.25$ & $5.5 \pm 0.57$ & $4.9 \pm 0.50$ & $6.2 \pm 0.48$ & $7.5 \pm 0.58$ & $5.3^{*} \pm 0.32$ & $11.0 \pm 0.30$ & $5.7 \pm 0.25$ \\
\hline \multicolumn{9}{|c|}{ Pneumonectomy } \\
\hline $\mathrm{FiO}_{2}(21 \%)$ & $1.5 \pm 0.12$ & $3.4 \pm 0.25$ & $3.1 \pm 0.24$ & $4.6 \pm 0.31$ & $5.5 \pm 0.88$ & $4.0 \pm 0.37$ & $9.3 \pm 1.65$ & $4.5 \pm 0.55$ \\
\hline $\mathrm{FiO}_{2}(5 \%)$ & $2.3 \pm 0.12$ & $5.1 \pm 0.14$ & $3.9 \pm 0.67$ & $5.8 \pm 0.75$ & $8.7 \pm 1.11$ & $7.1 \pm 1.30$ & $9.9 \pm 0.77$ & $4.8 \pm 0.60$ \\
\hline \multicolumn{9}{|l|}{ A-V Fistula } \\
\hline $\mathrm{FiO}_{2}(21 \%)$ & $2.2 \pm 0.12$ & $5.5 \pm 0.18$ & $4.7 \pm 0.50$ & $7.5 \pm 0.53$ & $8.9 \pm 0.95$ & $7.5 \pm 0.70$ & & \\
\hline $\mathrm{FiO}_{2}(5 \%)$ & $3.5 \pm 0.50$ & $8.6 \pm 1.10$ & $5.4 \pm 0.35$ & $8.6 \pm 1.00$ & $9.9 \pm 3.30$ & $8.4 \pm 2.70$ & & \\
\hline \multicolumn{9}{|c|}{$\begin{array}{c}\text { Pneumonectomy }+ \\
\text { A-V Fistula }\end{array}$} \\
\hline $\mathrm{FiO}_{2}(21 \%)$ & $2.6 \pm 0.09$ & $5.4 \pm 0.23$ & $7.4 \pm 1.41$ & $9.7 \pm 1.61$ & $7.8 \pm 2.73$ & $6.6 \pm 1.73$ & & \\
\hline $\mathrm{FiO}_{2}(5 \%)$ & $2.8=0.07$ & $5.8 \pm 0.20$ & $8.4 \pm 1.22$ & $11.5 \pm 1.09$ & $9.8 \pm 3.60$ & $8.3 \pm 2.23$ & & \\
\hline
\end{tabular}

\footnotetext{
${ }^{1}$ The figures represent mean and $S E$ of the mean.
} 


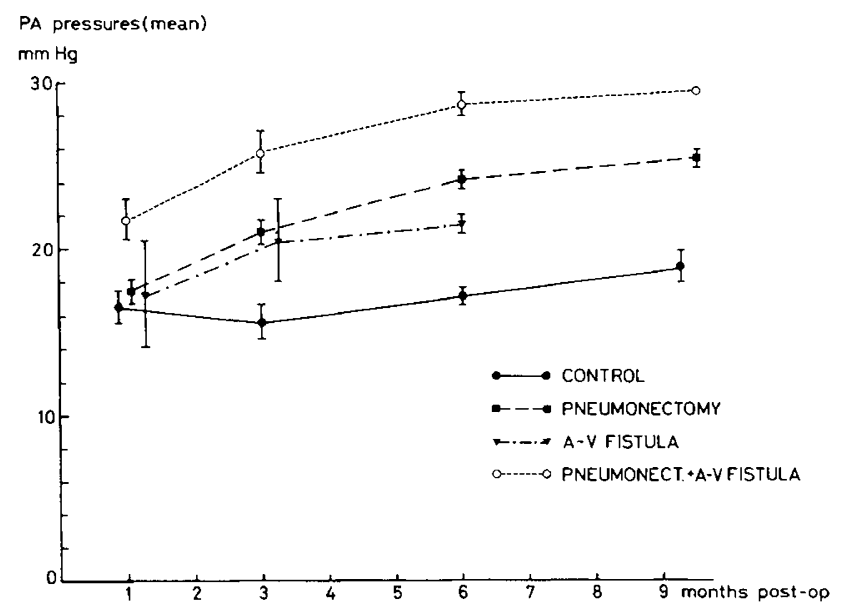

Fig. 1. Mean pulmonary artery (PA) pressures in the four groups during the 9 months follow-up. The vertical bars represent SE of the mean.

resistance was also increased, but to a lesser extent, because cardiac output was higher during hypoxia.

\section{EFFECT OF GLUCAGON ON REACTION TO HYPOXIA}

It has been reported that glucagon inhibits the pulmonary vascular response to hypoxia in calves (13). We administered glucagon $20 \mu \mathrm{g} / \mathrm{kg}$ body weight i.v. to four pigs. Two were controls, two had pneumonectomy, and all were 10 months old. The pigs were allowed to recover fully from the previous hypoxic vascular response before the injection. A further hypoxic episode was induced as before, 2-3 min after injection. The pressure response was unchanged after glucagon; mean PA pressure increase was the same before and after the injection in all four pigs (Fig. 2).

\section{HISTOLOGIC STUDIES (TABLE 3, FIG. 3)}

Medial wall thickness is shown in Figure 3 for all groups at the various stages of the study. In control pigs, the media of arterioles and small arteries was approximately $11 \%$ of the diameter and did not change up to 11 months of age (Fig. 4). Thus, the thickness of the media remains at a level similar to that in human neonates, and atrophy of the media, as seen in infants during the first year of life, does not occur. In pigs with a very high blood flow, i.e., pneumonectomy together with A-V fistula, relative wall thickness was much less in the first 6 months. Values around $6 \%$ were found (Fig. 5a). This was caused by marked dilation rather than to atrophy of the media. At 9 months postoperation, thickened small muscular arteries were found with relative wall thickness up to $21 \%$ (Fig. $5 b$ ), whereas other vessels in the same lung remained dilated. The result was a marked variability in wall thickness. The mean increased to reach the level found in control pigs, but the SD was greater. In pigs with pneumonectomy alone, medial wall thickness was also decreased as a result of dilation. The values were between those found in pigs with pneumonectomy plus fistula and the controls. No thickwalled vessels were found in the late follow-up studies.

Pigs with A-V fistula alone had medial wall thickness identical with those found in pigs with pneumonectomy alone; i.e., there was some dilation. No intimal changes occurred in the small arteries of any pig.

\section{RIGHT PULMONARY ARTERY (MAIN BRANCH)}

Control pigs had normal endothelium and some degree of diffuse metachromasia in the inner one-third of the media (Fig. $6 a$ ). In six pigs with increased flow, the amount of mucopolysaccharides in the media was increased, often forming little cystic spaces which disrupted the normal structure (Fig. 6b). Muscular hypertrophy was seen in the two pigs surviving to 9 months with

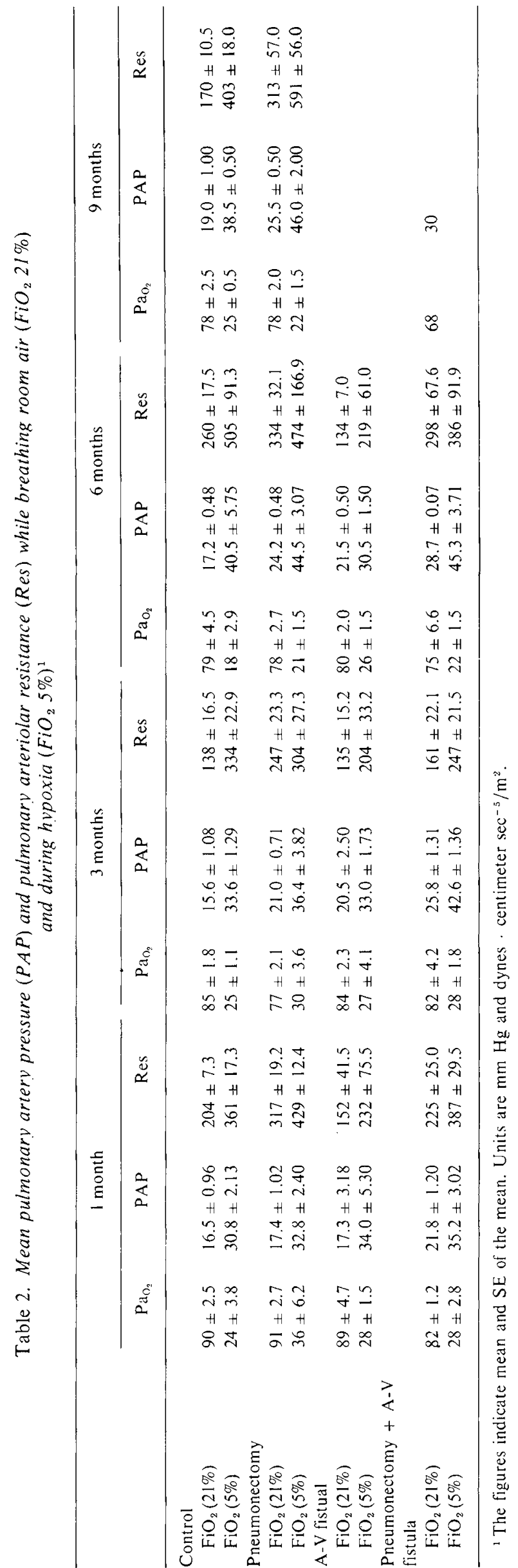


pneumonectomy and A-V fistula. Patches of intimal proliferation were prominent in three animals, one with A-V fistula only, one with pneumonectomy alone, and one with both $(6$ and 9 months after operation).

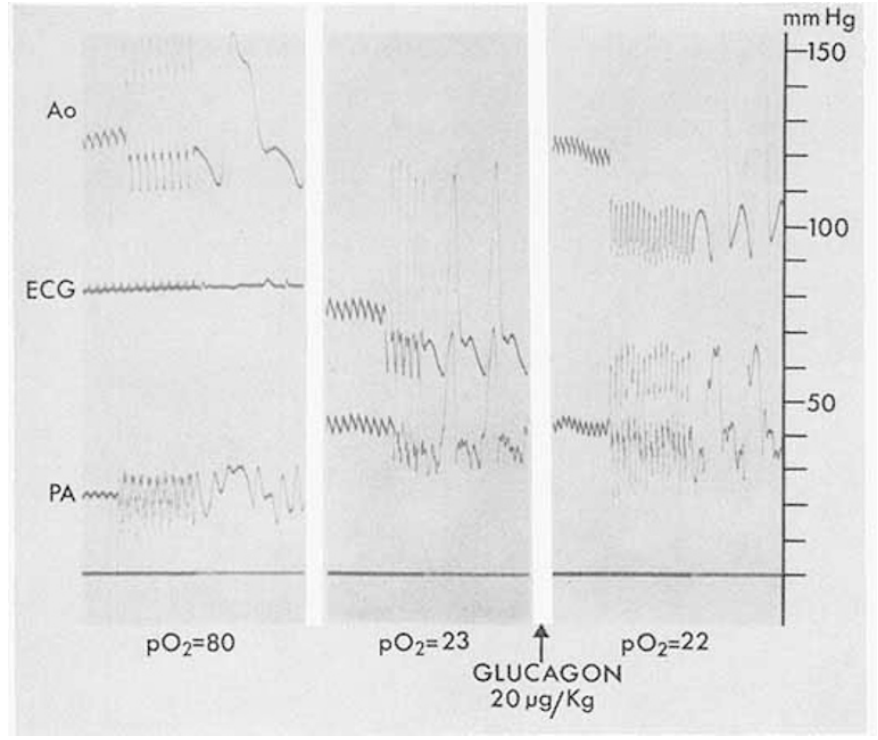

Fig. 2. Effect of glucagon on pulmonary vascular response to hypoxia in a 10-month-old pig. Pulmonary artery (PA) and aortic (AO) pressure curves on room air (left) and during hypoxia, before (middle), and after glucagon (right). The PA pressure response is identical. The drop in systemic pressure in this particular case is less after glucagon.

\section{DISCUSSION}

Among the models used in experimental research on pulmonary hypertension, those involving creation of an aortopulmonary fistula, especially end-to-end anastomosis, succeed best in producing lesions resembling pulmonary vascular disease $(1,4)$. However, in such models, the effect of individual factors, i.e.. increased flow, high pulmonary artery pressure, and increased left atrial pressure cannot be studied; indeed, two, if not all three, factors are produced as a result of the operation. To study the effect of increased flow alone, "obligatory" shunts, not connecting systemic and pulmonary arteries, are best created. We have chosen to

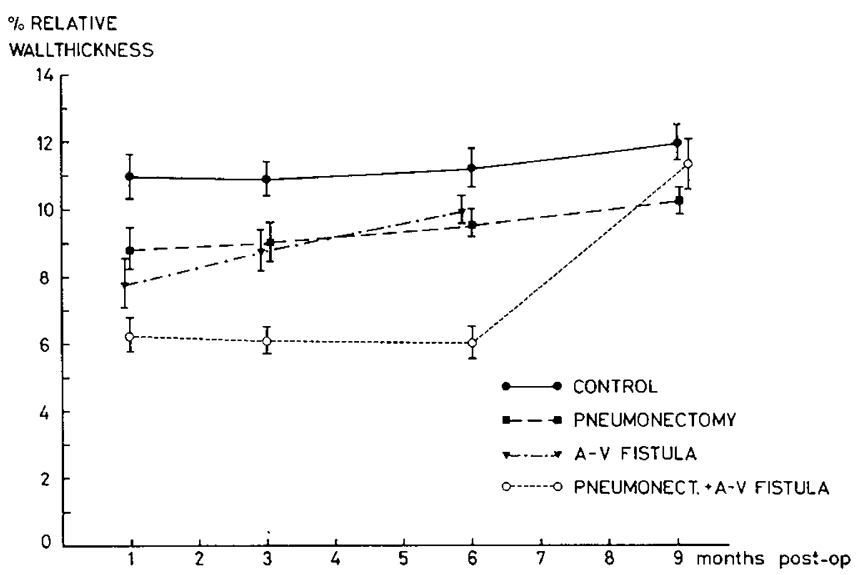

Fig. 3. Relative thickness of the media of small arteries and arterioles, in percentage of the arteriolar diameter (measured at the level of the external lamina elastica). The vertical bars represent SE of the mean. See text.

Table 3. Relative wall thickness of small arteries

\begin{tabular}{|c|c|c|c|c|}
\hline & 1 month, \% & 3 months, $\%$ & 6 months, $\%$ & 9 months, $\%$ \\
\hline Control & $11.0 \pm 0.67$ & $10.9 \pm 0.51$ & $11.2 \pm 0.61$ & $12.0 \pm 0.57$ \\
\hline Pneumonectomy & $8.8 \pm 0.65$ & $9.0 \pm 0.63$ & $9.6 \pm 0.40$ & $10.3 \perp 0.37$ \\
\hline A-V fistula & $7.8 \pm 0.74$ & $8.8 \pm 0.58$ & $9.9 \pm 0.46$ & \\
\hline Pneumonectomy $+\mathrm{A}-\mathrm{V}$ fistula & $6.3 \pm 0.47$ & $6.1 \pm 0.40$ & $6.1 \pm 0.44$ & $11.3 \pm 0.73$ \\
\hline
\end{tabular}
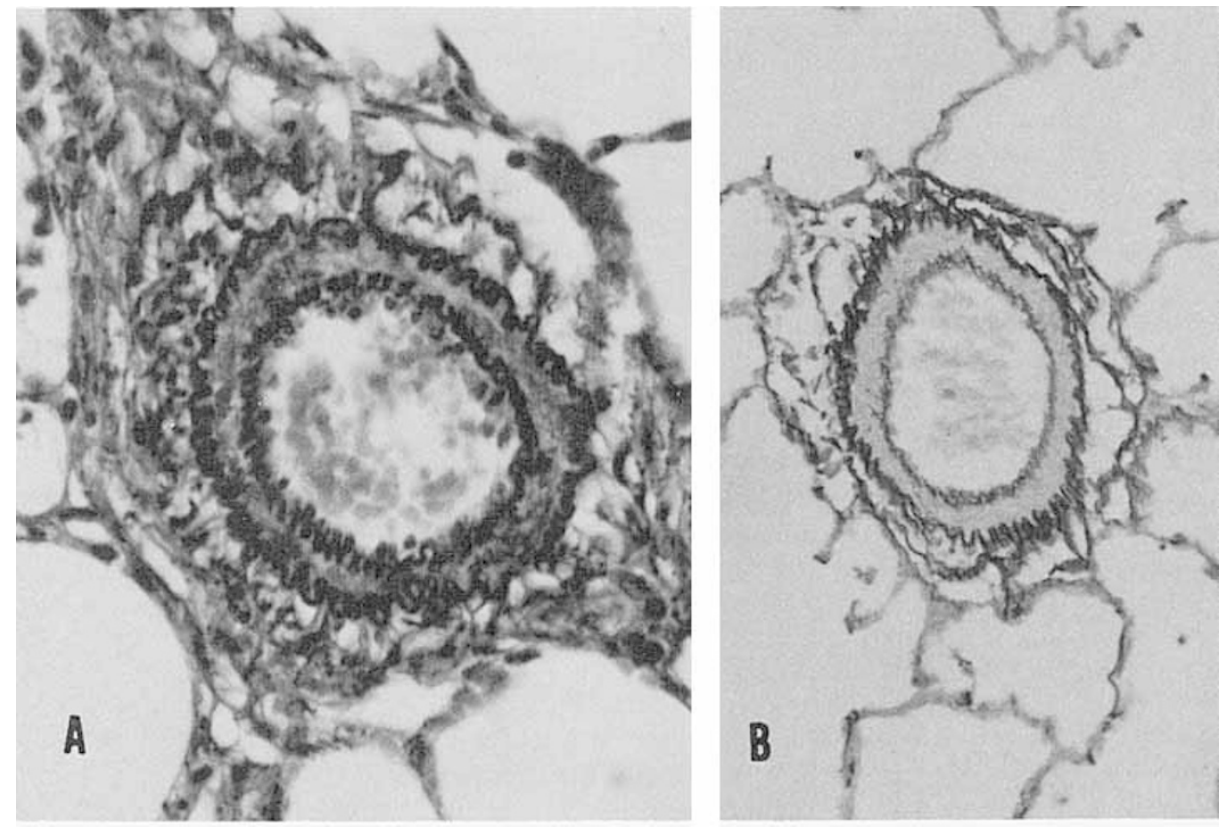

Fig. 4. Smail muscular pulmonary arteries in control pigs at the age of 2 months $(a)$ and 10 months $(b)$. No atrophy of the media occurs. Elastic-van Gieson, $\times 320$. 

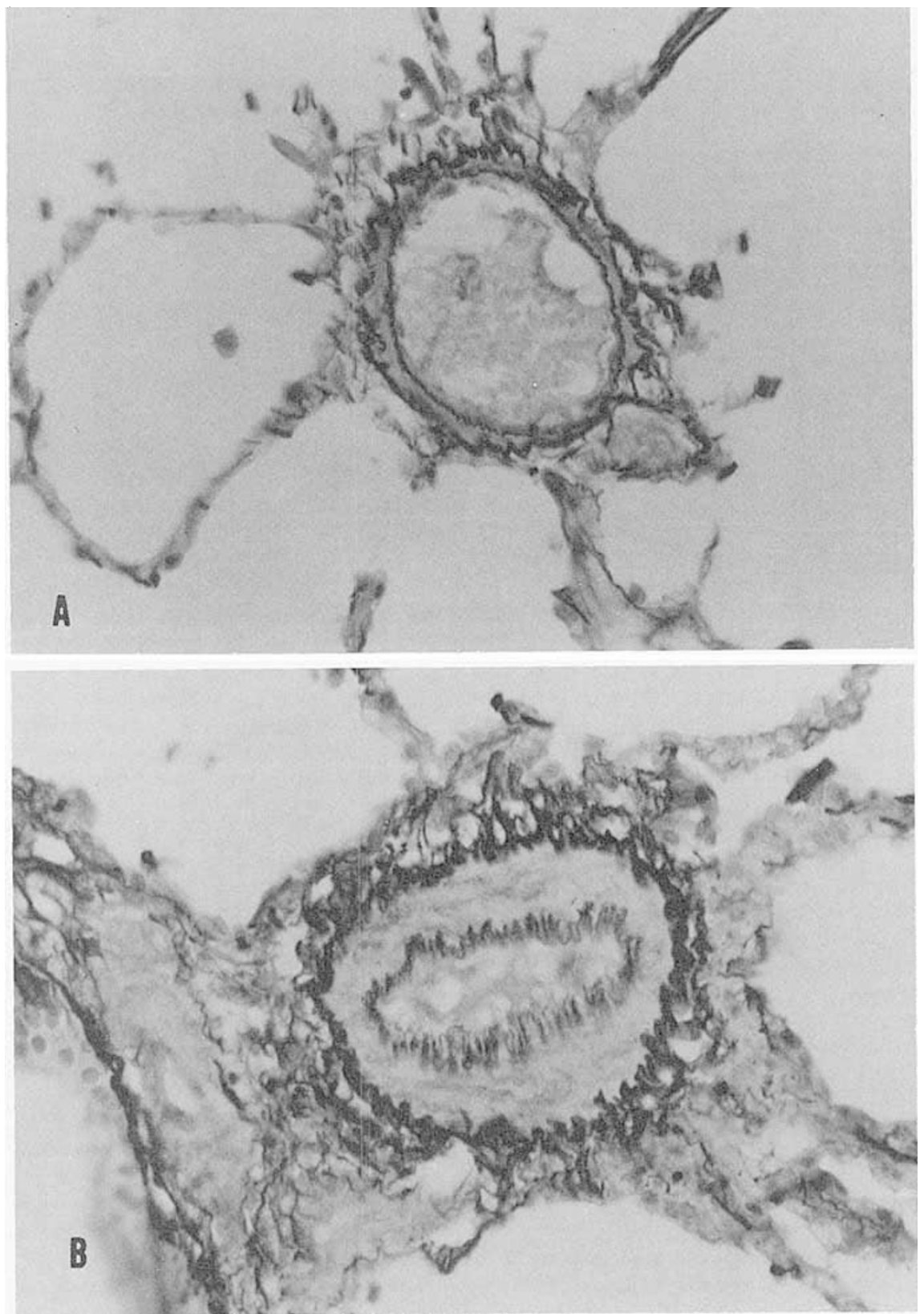

Fig. 5. a: pulmonary arteriole in a pig with A-V fistula and pneumonectomy 3 months after surgery. There is marked dilation of the vessel with thinning of the wall. $b: 9$ months after pneumonectomy and construction of an A-V fistula, some thick-walled arterioles can be seen. Elastic-van Gieson, $\times 320$.

construct A-V fistulas, combining them with left pneumonectomy. Pneumonectomy diverts the whole of the cardiac output to the remaining lung, whereby the pulmonary blood flow on that side is almost doubled. The A-V fistulas we created in the neck vessles remained open in most animals throughout the study period: they increased cardiac output, on the mean, by about $70 \%$. Therefore, pigs with fistulas and pneumonectomy had a slightly more than threefold increase in pulmonary blood flow to the remaining lung. Despite this, left atrial pressure was not significantly increased and PA pressure was only slightly higher than in control animals at the onset of the study; therefore, at least early in the experiment, increased blood flow was the only pathogenic factor produced by this model.

In the control pigs, PA pressure was low (mean $15-19 \mathrm{~mm} \mathrm{Hg}$ ) throughout the study; no change occurred between the ages of 2 months (first study) and 11 months (last study). This indicates that "adult" PA pressure was reached at the age of 2 months. The stable PA pressure correlates well with the histologic aspect of the small pulmonary arteries; relative medial wall thickness remained unchanged and was $11-12 \%$ of the vessel diameter up to almost 1 year of age. This is the thickness found in human neonates $(5,14)$. However, in the human, atrophy of the medial muscle coat occurs in the first months of life, and the relative medial wall thickness will decrease to about $35 \%$. In this respect, the pulmonary circulation of piglets differs from the human, a fact that has been mentioned before (14). This also explains why the pulmonary circulation remained highly reactive to hypoxia throughout the study. The increase in PA pressure in response to 5\% oxygen breathing was as marked at 11 as it was at 2 months of age. Because of this reactivity, it was important to maintain good ventilation and monitor blood gases closely. Any drop in $\mathrm{P}_{(\mathrm{O})}$, below $65 \mathrm{~mm} \mathrm{Hg}$ resulted in an increase in PA pressure. $\mathrm{pH}$ also had to be kept constant, as it has been shown that acidosis may increase PA pressure (11).

In the pigs which were operated on, a progressive increase in PA pressure could be observed. The amount of increase appears to be directly related to flow as it was more marked in pigs with pneumonectomy plus A-V fistulas, than in those with either one of 

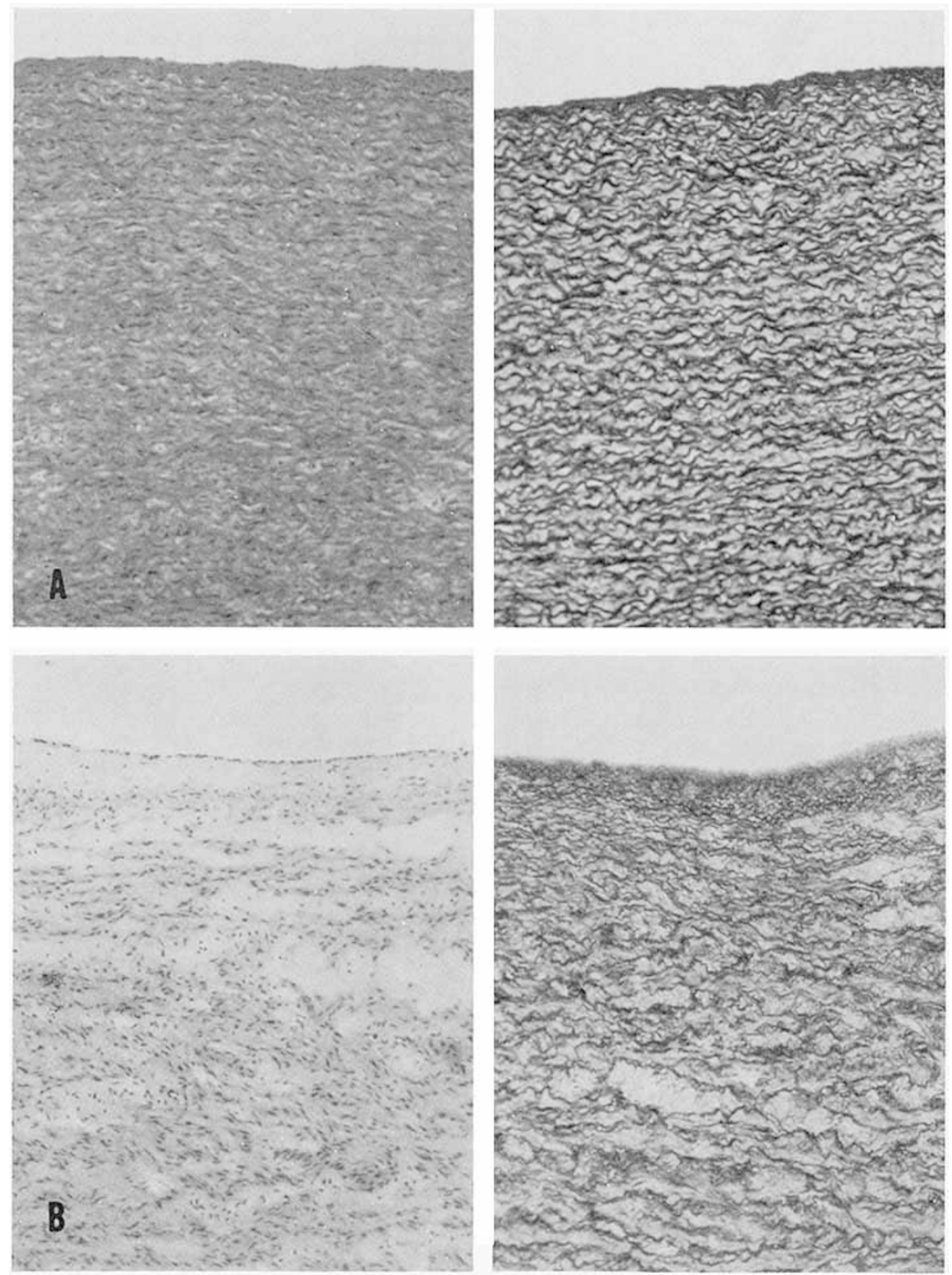

Fig. 6. a: right pulmonary artery in a 7-month-old control pig. Note regular arrangement of the elastic tissue and absence of intimal thickening. Left: hematoxylin-eosin; right: elastic-alcian blue, $\times \quad 50 . b$ : right pulmonary artery in a pig with high pulmonary blood flow, 9 months after pneumonectomy and construction of an A-V fistula. Note irregular arrangement of the elastic tissue and cystic spaces filled with mucopolysaccharides. There is thickening of the intima. Left: hematoxylin-eosin; right: elastic-alcian blue, $\times 50$.

these lesions. In the group with the combined operation, mean PA pressure was slightly (but significantly) higher than control at 1 month after surgery already. At 3 months, the difference was more important; by then, a significant rise in PA pressure had occurred in the groups with only one lesion as well. A further increase was noted in the following months. Previous studies in our laboratory (7) and by others (10) have already shown that an increase in PA pressure may occur after pneumonectomy in certain laboratory animals. Rudolph et al. (10) observed a PA pressure rise in most of his pneumonectomized dogs, however, Kato et al. (7) were unable to demonstrate the same in 25 puppies; they did see a PA pressure rise in pneumonectomized minipigs.

As for A-V fistulas, a previous study by Hawe et al. (6) suggests that they induce a very slight rise in PA pressure of dogs. Our studies indicate that the two lesions combined add up their effect on PA pressure, so that there is a good correlation between the amount increase in pulmonary blood flow, and the degree of elevation of PA pressure. This appears to be "hyperdynamic" pulmonary hypertension. Resistance data, however, require careful interpretation in our model. Pneumonectomy itself increases resistance regardless of the state of pulmonary arterioles by decreasing, by almost half, the number of capillaries and arterioles. Mair et al. (8) therefore suggest that, when there is only one PA branch, calculated resistance should be divided by 2 if one wants to make any conclusions on the state of the pulmonary vascular tree, i.e., the amount of pulmonary vascular disease present. If this is done in our study, resistances markedly lower than control will be found in all piglets with pneumonectomy, especially those with additional A-V fistulas, in the first 3 months. Only after 6-9 months is there some increase in resistance in these groups; the actual values, however, do not exceed those of control animals (still considering the halved values for animals with pneumonectomy).

Another parameter often used in clinical studies on pulmonary vascular disease, the resistance ratio (pulmonary resistance divided by systemic resistance), is even more difficult to interpret; indeed, 
A-V fistulas markedly lower the systemic resistance. Because of these problems, we believe that PA pressure is the physiologic parameter best used to make comparison between groups in our model.

The main histologic finding in arterioles of pigs with increased pulmonary blood flow was thinning of the muscular media, not by atrophy, but by marked dilation. Here again, the changes correlated with flow, as they were significantly more marked in piglets with both pneumonectomy and A-V fistula than in animals with only one lesion. Such dilation of the vessels was also noted to occur in puppies with pneumonectomy (10), as demonstrated by angiography. In our pigs with pneumonectomy alone or A-V fistulas this remained the only change in arterioles thoughout the study.

By contrast, those pigs with the highest blood flow (pneumonectomy and fistula) did develop thickened arterioles, which were found scattered among dilated ones. Extrapolating from clinical correlates, one could expect that pulmonary vascular changes would be more prominent in pigs with pneumonectomy plus A-V fistulas. Indeed, Pool's extensive study (9) on unilateral absence of a pulmonary artery shows that pulmonary vascular disease occurs in a small percentage when this lesion is isolated, but in a very high percentage when a left-to-right shunt is associated. Also, infants with a ventricular septal defect have a high risk of developing pulmonary vascular disease early in life if their pulmonary blood flow equals or exeeeds by 3 times the systemic blood flow (2). Pigs with combined pneumonectomy plus $\mathrm{A}-\mathrm{V}$ fistula had pulmonary blood flows in this range.

The appearance of thick arterioles may be surprising as it has been stated that severe pulmonary hypertension is necessary to create medial muscular hypertrophy (15); high flow is believed to cause intimal changes, at least in the acute experiment (3). Such intimal changes did not occur in small muscular arteries, but they were seen in the right pulmonary artery of several pigs with high flow. These large elastic arteries did, in fact, show most of the changes seen in human pulmonary hypertension: muscular hypertrophy, changes in the ground substance including small cystic spaces filled with metachromatic material, and patchy intimal fibrosis.

Most of these changes, and particularly medial hypertrophy, have been ascribed, at least by some (15), to high intravascular pressure rather than increased flow. However, on theoretic grounds, they are not unexpected. Indeed, the pathogenetic factor likely to cause most of the changes is arterial wall tension rather than actual intravascular pressure. Wall tension depends on diameter of the vessel as well as on pressure, according to Laplace's law (5). When the whole of the pulmonary vascular bed is dilated because of high flow, and the diameter of the vessels is therefore large, even a slight to moderate increase in pressure will increase wall tension considerably, creating the stimuli for hypertrophy in muscular arteries and changes in the wall of elastic arteries. We believe that this is the sequence of events in our experimental model; it may well explain the development of certain features of pulmonary vascular disease secondary to high flow with only mild or moderate increase in PA pressure and a normal left atrial pressure. The clinical correlate, in the human pulmonary circulation, is unilateral absence of the pulmonary artery; that pulmonary vascular disease may occur in these patients has been shown by Pool (9). High flow is again the main, if not the only, physiologic factor involved. Of course, high PA pressure will constitute an aggravating factor, as it further increases wall tension. Thus, it is not surprising that experimental models creating high flow and high pressure result in more severe and earlier vascular lesions. However, we may conclude from the present study that severe elevation of pressure is not a prerequisite for the appearance of changes in the pulmonary arteries and arterioles.

\section{SUMMARY}

A model of high pulmonary blood flow was created in piglets by left pneumonectomy, arteriovenous fistulas in the neck, and a combination of both. The pulmonary blood flow was thereby increased up to 3 times above control values. A slight to moderate increase in pulmonary artery pressures resulted, most marked in the group with the highest flow.

The small pulmonary arteries and arterioles showed marked dilation in response to high flow, with a decrease in relative wall thickness. In the group with the highest flow, thick-walled arterioles appeared between 6 and 9 months follow-up. The right pulmonary artery showed patches of intimal thickening and changes in the media. Thus, some signs of pulmonary vascular disease appeared in this high flow model despite only slight increase in pulmonary artery pressure.

\section{REFERENCES AND NOTES}

1. Ferguson, D. J., Berkas, E. M., and Varco, R. L.: Experimental methods for the production of pulmonary hypertension. In: W. Adams and I. Veith: Pulmonary Circulation. (Grune and Stratton, New York, 1959).

2. Friedli, B., Kidd, B. S. L., Mustard, W. T., and Keith, J. D.: Ventricular septal defect with increased pulmonary vascular resistance. Amer. J. Cardiol., 33: 403 (1974).

3. Fry, D. L.: Acute vascular endothelial changes associated with increased blood velocity gradients. Circ. Res., 22: 165 (1968).

4. Gupta, S., Maitra, T. K.. Viswanathan. S., and Basu, A. J.: An experimental model of secondary pulmonary hypertension. J. Thorac. Cardiovasc. Surg., 64: 953 (1972).

5. Harris, P., and Heath, D.: The Human Pulmonary Circulation (Livingstone, Ltd., London, 1962).

6. Hawe, A., Tsakiris, A. G., Rastelli, G. C., Titu, J. L., and McGoon, D. C.: Experimental studies of the pathogenesis of pulmonary vascular obstructive disease. J. Thorac. Cardiovasc. Surg., 63: 652 (1972).

7. Kato, H.. Kidd, B. S. L., and Olley, P. M.: Effects of hypoxia on pulmonary vascular reactivity in pneumonectomized puppies and minipigs. Circ. Res., 28: 397 (1971).

8. Mair, D. D., Ritter, D. G., Davis, G. D. Wallace, R. B., Danielson, G. K., and McGoon, D. C.: Selection of patients with truncus arteriosus for surgical correction. Circulation, 49: 144 (1974)

9. Pool, P. E., Vogel, J. H. K., and Blount, S. G.: Congenital unilateral absence of a pulmonary artery. Amer. J. Cardiol., 10:706 (1962).

10. Rudolph, A. M., Neuhauser, E. B. D., Golinko, R. J., and Auld, P. A. M.: Effects of pneumonectomy on pulmonary circulation in adult and young animals. Circ. Res., 9: 856 (1961).

11. Rudoiph, A. M., and Yuan, S.: Response of the pulmonary vasculature to hypoxia and $\mathrm{H}^{-}$ion concentration changes. J. Clin. Invest., 45: 399 (1966).

12. Seuffert, R. W., Giese, R., and Meyer, R.: Bestimmung der Meeh'schen Konstante bei Haustieren. Beitr. Physiol., 3: 203 (1926).

13. Spangler, R. D., and Grover, R. F.: Attenuation of hypoxic pulmonary hypertension in newborn calves by glucagon (Abstract.) Amer. J. Cardiol., 31 : 159 (1973).

14. Wagenvoort, C. A., Heath, D., and Edwards, J. E.: Pathology of the Pulmonary Vasculature (Charles C Thomas, Publisher, Springfield, Ill., 1964).

15. Wagenvoort, C. A., Nauta, J., Van der Schaar, P. J., Weeda, H. W. H., and Wagenvoort, N.: Effect of flow and pressure on pulmonary vessels. Circulation, 35: 1028 (1967).

16. We wish to thank Drs. W. Zingg and Lee Wai-Tsun for their invaluable surgical help in performing pneumonectomies and arteriovenous fistulas. Gratitude is expressed to Dr. M. Silver for his advice in interpreting the histologic findings and to Mrs. E. Rapaport for preparing the sections.

17. Requests for reprints should be addressed to: B. Friedli, M.D., Clinique Universitaire de Pediatrie, Hôpital Cantonal, 1205 Geneva (Switzerland). 18. Accepted for publication March 1I, 1975. 